\title{
Lane Detection and Tracking Algorithm Based on Curve Fitting Model
}

\author{
Rajakumar.R ${ }^{\mathrm{a}, 1}$, Pandian. $\mathrm{R}^{\mathrm{b}}$, and PremJacob. $\mathrm{T}^{\mathrm{c}}$, \\ ${ }^{a} U G$ Scholar, Dept of ECE, Sathyabama Institute of Science and Technology, Chennai, \\ India \\ ${ }^{b} U G$ Scholar, Dept of ECE, Sathyabama Institute of Science and Technology, Chennai, \\ India \\ ${ }^{c} U G$ Scholar, Dept of ECE, Anna University, MIT Campus, Chennai, India
}

\begin{abstract}
Lane Detection is the fundamental vehicle driving system and selfdriving. The proposed concept is to employ the pixel difference in the expected lane and the road backdrop to detach the lane from the road, and then the Curve fitting model is made use of in the segregated lanes to locate the straight line in the image as the lane line. This paper offers a lane detection method based on the Sobel filter and Curve-fitting model for lane line tracking in different conditions. The main objective is to improve the accuracy of the Xi'an city database and the KITTI vision benchmark suite dataset. To achieve this HLS color space was performed which identifies the lane by adding pixel values.
\end{abstract}

Keywords. Sobel filter, Curve fitting model, Lane detection, Lane tracking, sliding window, deep neural network.

\section{Introduction}

Most accidents occur due to invisible road lanes. The accidents can be reduced drastically, by employing improved driving assists. A system that warns the driver, can save considerable number of lives..Indriving assistance to achieve safety on roads, the challenging tasks are road lanes' detection or boundaries' detection which is exposed in white and yellow lines on roads. In this work, we developed a Curve fitting model improving the robustness of detecting and tracking the lane for safe transportation.In our method, inspection of lane and its tracking will be done by the Curve fitting model and related component function to improvise the working of the lane detection and tracking.

\section{Related Works}

The literature[1] extracted the Adaptive Region of Interest to minimise computational complexity. Then Kalman filter is used to find road boundaries sensed in the AROIusing Progressive Probabilistic Hough Transform (PPHT) in the succeeding frame. 
In literature [2] lane marks in road images are extracted which is based on the multiconstraints model and a clustering algorithm based is proposed to detect the lane. The literature [3] used the B-spline fitting lane line from the strength of the RANSAC algorithm for detection offront-view images and improved Hough transform to find rear-view images. The literature [4] improved the accuracy of lane recognition and reduced the difference between pixels. The literature [6] proposed an flexible road detection technique that connects both lane lines and boundaries of obstacle , applicable for detecting lanes. The literature [7] makes use of a color threshold method to identify lane edges with perspective transformations and Hough transform to find out lane segments in the image with measured conditions. These conditions comprise a straight road and a sunny weather. In literature [8] based on the Vision-Based Extrapolation method which performs well in controlled weather conditions and Hough transform used to detect only the straight lane. In this paper just as in [5] edge-based detection with the open street map is used to detect lanes which increases computation time. Hough Transform [9] is employed to identify the straight lane and the Curve fitting model detects the curve lane which increases the computation time [13-14].

\section{Lane Detection Algorithm}

Lane detection in images is a significant operation in image processing and computer vision. Lane detection algorithms detect the lane edges and determine the vehicle position in the lane. A single monocular camera is used for lane detection. This work corresponds to the accurate location of the vehicle in the same lane. This system recognizes the majority of white and yellow markings across the lane effectively during different conditions which include shadows, rain, snow, or other disturbances on the road. The preprocessed image is perspective transformed which converts a $3 \mathrm{D}$ image to a 2Dimage.Then the Sobel filter was performed for noise reduction and to identify the pixel representing the edge. The sliding window method is applied from the bottom of the image by identifying lane pixels. To calculate the vehicle's center offset, the polynomial fit in pixels is used to determine the $\mathrm{x}$ position of the left and right lane corresponding to the $y$ at the bottom of the image.

\subsection{Sobel Edge Detection}

By applying the threshold a particular part of the image, ROI, we have an HLS color image as input. In this step, to locate boundaries of lane, one edge detection method called the Sobel filter, is used and the boundaries are detected. In Sobel filter, the main objective is to detect the edges that are nearer to the real lane edges. Sobel edge detection basically uses the gradient vector of an intense image. The captured color image is transformed to HLS color space to make the method quicker, computationally less intensive, and less sensitive to scene conditions. To detect the white lane, lightness is set to a value close to $100 \%$. Then the combination of saturation and lightness value was defined to detect the yellow lane. In our proposed method, captured images choose from the directory of the KITTI Benchmark suite, would be processed. The camera is so calibrated that the vanishing point of the road should be kept on the top of the Region of Interest(ROI). 


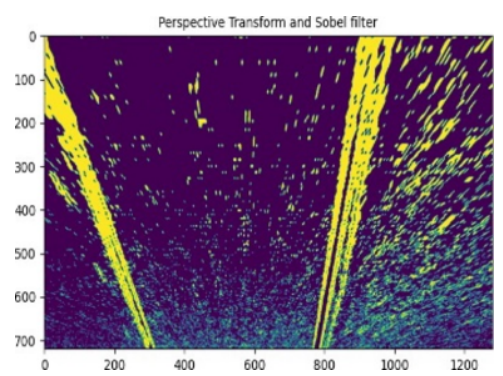

Figure 1.Sobel filter and Perspective transform

\subsection{Lane Detection and Tracking Flow Chart}

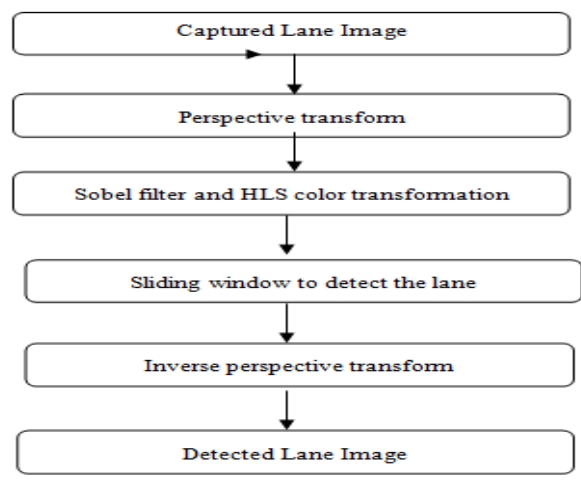

Figure 2. Lane detection and tracking flow chart

\section{Lane Tracking algorithm}

Lane Tracking is mainly employed to increase the computation efficiency of the lane detection algorithm by keeping the earlier information of how the states have an estimateofthefuturestates. Thisalgorithmincludesapredictionstepas well as a measurement step. In the case of Lane Tracking, the prediction stage involves shifting the detected lanes by a specific amount in the image, based on polynomial fit. In the measurement step, the radius of curvature and vehicle offset were computed.

\subsection{Sliding Window}

To draw multiple sliding windows, the starting point of the windows should be known. To find the initial point, a histogram for the bottom part of the image is calculated. Based on the peak value of the histogram, the initial window is selected and the mean of the non-zero points inside the window is determined. For the first half of the image, the left lane peak is obtained and the other right half gives the peak of the right lane. Thus, left and right starting sliding windows are formed, and then left lane center and right lane center are calculated. This kind of selection works fine for both lanes are in the left 
lane on the left side of the image and the right lane on the right side of the image.The sliding window output is in figure 3.

\subsection{Lane Design Parameters}

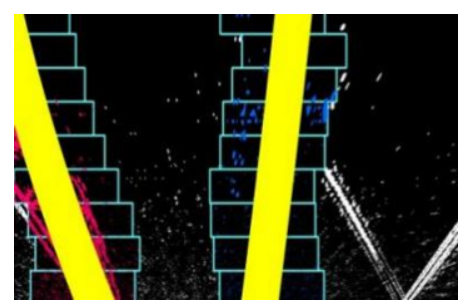

Figure 3.Sliding window output

The equation of the curve model is given as,

$$
\mathrm{Ax}{ }^{2}+\mathrm{Bx}+\mathrm{C}=0
$$

As A, B, C are the constants of the quadratic curve, five of which three constants of the quadratic curve are thus state radius of the curvature is calculated using the $y$ axis point at the bottom of the image.

$$
R=\left(1+\left(d^{2} y / d x^{2}\right)\right)^{3 / 2}(d y / d x)^{2} d x, d y \text { are the lane positions. }
$$

\section{Experimental Results}

\subsection{Lane Detection For Video Frames From Xi'an Citydataset}

The present section details the experimental out comes of our method of detection of lane with two sets of various video frames obtained from the Xi' an city dataset.

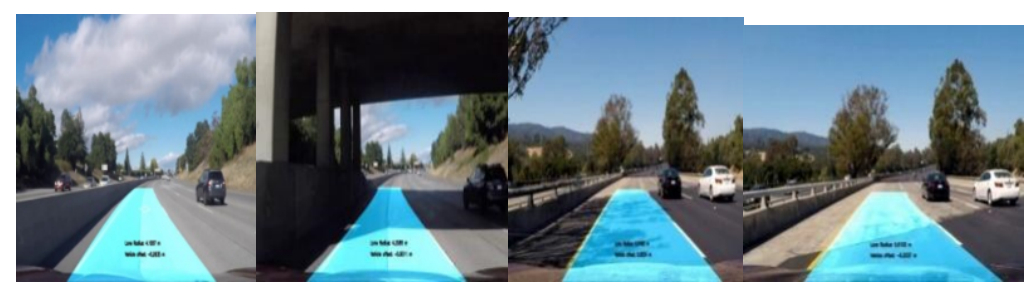

Figure4.a,b,c,d Output frame of Xi' an city database

\subsection{Radius Calculation Of Xi'an City Database}

The radius and vehicle offset ofa lane in the Xi'an city video database calculated using the equation 2 formula and shown in table 1. 
Table 1. Radius and Vehicle offset calculation of Xi' an city database

\begin{tabular}{lll}
\hline IMAGE & RADIUS (METER) & VEHICLE OFFSET (METER) \\
\hline Fig.5.a & 4.1007 & -0.263 \\
Fig.5.b & 4.3386 & -0.001 \\
Fig.5.c & 6.6480 & 0.0834 \\
Fig.5.d & 6.6102 & -0.2037 \\
\hline
\end{tabular}

\section{Conclusion and Future Work}

Lane detection was done using the HLS color space and edge detection by using the Sobel filter. Then curve fitting model was used for efficient lane detection and tracking. For two video frames accuracy is calculated as $94.80 \%$ and for the KITTI dataset (not described inside this paper) accuracy was calculated as $95.83 \%$.This algorithm computation time was calculated as (150-200milliseconds/frame) which is suitable for real-time applications. This algorithm can be further developed for self-driving vehicles.

\section{References}

[1] MehrezMarzougui, Areejalasiry, Yassinkortli and JamelbailI ,A Lane Tracking Method Based on Progressive Probabilistic Hough Transform, IEEE access vol.8 pp. 84893 - 84905, 13 May 2020.

[2] HanyuXuan, Hongzhe Liul, Jiazheng Yuan, And Qing Li, Robust Lane-Mark ExtractionforAutonomousDrivingUnderComplexRealConditions,IEEEAcess,Vol. 6, pp.5749-5766, March 9, 2018.

[3] HuiXiong;DamengYu;JinxinLiu;HeyeHuang;QingXu;JianqiangWang;Keqiang Li, Fast and robust approaches for lane detection using multi-camera fusion incomplex scenes, IET Intelligent Transport Systems, Volume: 14, Issue: 12, 12 2020), pp. 1582-1593, 19 November 2020.

[4] Xingyao Wang, Da Yan, Ke Chen, Yancong Deng, Cheng Long, Kunlin Zhang, Sibo Yan, Lane Extraction and Quality Evaluation: A Hough Transform Based Approach, 2020 IEEE Conference on Multimedia Information Processing andRetrieval (MIPR), September 03, 2020.

[5] Guanqi Ding; Xiansheng Qin, Vision-based Edge-lined Assisted Moving Vehicle Detection, 2020 IEEE International Conference on Mechatronics and Automation (ICMA), 13-16 Oct. 2020.

[6] Jing LiJunzheng Wang; Min Yan, Adaptive road detection method combining lane line and obstacle boundary, IET Image Processing, Volume: 14, Issue: 10, pp. 2216 - 2226, 15 October 2020.

[7] StevanStević, Marko Dragojević, MomčiloKrunić, NenadČetić, Vision-Based Extrapolation of Road Lane Lines in Controlled Conditions, 2020 Zooming Innovation in Consumer Technologies Conference (ZINC), August 15, 2020.

[8] Xiaoliang Wang; YeqiangQian; Chunxiang Wang; Ming Yang,Map-Enhanced Ego-Lane Detection in the Missing Feature Scenarios, IEEE Acess, vol.8, pp. 107958 - 107968, 8 June 2020.

[9] Huifeng Wang, Yunfei Wang, Xiangmo Zhao, Guiping Wang, He Huang, Jiajia Zhang, Lane Detection of Curving Road for Structural High-way with Straight-curve Model on Vision , IEEE Transactions on Vehicular Technology vol.68 issue:6 pp. 5321-5330,26 April 2019.

[10] Lim.K.H, et al, Lane detection and Kalman-based linear parabolic lane tracking, International Conference on Intelligent Human-Machine Systems and Cybernetics .2009. pp.351-354.

[11] Deng.J, and Han .Y. A real-time system of lane detection and tracking based on optimized RANSAC B-spline fitting. Research in Adaptive and Convergent System, 2013, pp. 157-164.

[12] Gopalan .R, Shneier.M, et al, A learning approach toward detection and tracking of lane markings. IEEE Trans on International Transportation System, vol. 13,no. 3,pp. 1088-1098, Mar. 2012.

[13] Ambeth Kumar.V.D ,.Malathi.S, Ashok Kumar.V.D (2015) .Performance Improvement Using an Automation System for Segmentation of Multiple Parametric Features Based on Human Footprint . for the Journal of Electrical Engineering \& Technology (JEET) , vol. 10, no. 4, pp.1815-1821 .2015. [http://dx.doi.org/10.5370/JEET.2015.10.4.1815] 
[14] V.D.Ambeth Kumar et.al,, "An Efficient Security System for Data base Management from Illegal Access, IEEE International Conference on Wireless Communications, Signal Processing and Networking, 2016. DOI: 10.1109/WiSPNET.2016.7566252 